\title{
MOTION BASED ACTION RECOGNITION USING k-NEAREST NEIGHBOR
}

\author{
Shikha.A.Biswas ${ }^{1}$, Vasant.N.Bhonge ${ }^{2}$ \\ ${ }^{1}$ M.E. Student, Branch Digital Electronics, Department of Electronics and Tele-communication, Shri Sant Gajanan \\ Maharaja College of Engineering, Shegaon, Sant Gadge Baba Amravati University, Maharashtra State, India. \\ ${ }^{2}$ Associate Professor, Branch Digital Electronics, Department of Electronics and Tele-communication, Shri Sant \\ Gajanan Maharaja College of Engineering, Shegaon, Sant Gadge Baba Amravati University, Maharashtra State, \\ India.
}

\begin{abstract}
Analyzing the actions of a person from a video by using computer is termed as Action Recognition. This is an active research topicin the area of computer vision. There are many applications of this research which include surveillance systems, patient monitoring systems, human performance analysis, content-based image/video retrieval/storage, virtual reality. Although many efficient applications are available of action recognition, the most active application domain in the area of computer vision is to "look at people". In this paper, motion feature is extracted because motion features can portray the moving direction of human body and hence human actions can be effectively recognized by motion rather than other features such as color, texture or shape. In the motion-based approach, the method that extracts motion of the human action such as motion blob, optical flow, FIRfiltering or watershed transform are used for recognizing action. This paper presents a novel method of action recognition that analyzes human movements directly from video. The overall system consists of major three steps: blob extraction, feature extraction and action recognition. In the first step, the input video is preprocessed to extract the $2 \mathrm{D}$ blob. In the second step, motion feature is extracted using optical flow and at last action is recognized using classifier $k$-Nearest Neighbor (kNN).
\end{abstract}

Keywords: Action Recognition, 2Dblob, Optical Flow, kNN

\section{INTRODUCTION}

Recognizing or understanding the actions of a person from a video is the objective of action recognition. The main objective of our method is to improve the accuracy of recognition. Action recognition is classified into four types: Object-level, Tracking-level, Pose-level \& Activity-level. Object-level recognize the locations of object, Tracking-level recognize the object trajectories,

Pose-level recognize the pose of a person \& Activity-level recognize the activity of person.The major problems faced in action recognition are: view-point variation: movement of camera, temporal variation: Variation in duration and shift, spatial variation: Different people perform the same action in different ways.n our paper we present a method that eliminates all the above problems by using the concept of optical flow and kNN.

\section{TOOL}

The tool used for recognition is MATLAB, version 7.10.0(R2010a)

\section{DATASETS}

The datasets used for Action Recognition are KTH and Weizmann. [9], [12]

\section{Weizmann}

It contains 10 types of actions performed by 9 subjects. Thus it contains total 90 AVI videos, taken with a static camera and static background and with a frame rate of 25 fps. Actions in this dataset include: bend, jack, run, side, skip, wave1, wave2, jump, P-jump walk.

\section{$\underline{\mathrm{KTH}}$}

It contains 6 types of actions performed by 25 subjects in 4 homogenous backgrounds. Thus it contains total 600 AVI videos, taken with a static camera over homogenous backgrounds \& with a frame rate of $25 \mathrm{fps}$. Actions in this dataset include: walking, jogging, running, boxing, hand waving, and hand clapping.

\section{METHOD}

The proposed method contains three main stages of recognition:

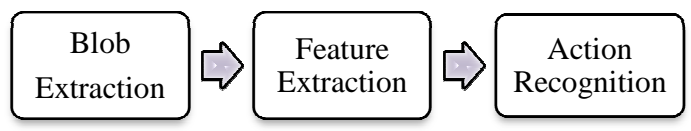

Fig-1: Stages of Action Recognition 


\subsection{Blob Extraction}

The most commonly used low-level feature for identifying human action is $2 \mathrm{D}$ blob. Hence the first stage is called blob extraction or segmentation or pre-processing stage In this stage, the color video is first converted from RGB to gray and then finally to binary. To remove the salt and pepper noise, the gray scale video is first median filtered and then is converted into binary using autothresholding.

Thus this stage divide the input video into two classes i.e. foreground (activity of the human) called the 2D blob, and background (empty frame). Then for enhancement, dilation is done. In the dilation process, the binary video is dilated by a structuring element (called mask or window) of size $3 \mathrm{x}$ $3,5 \times 5$ or $7 \times 7$.The structuring element of the proposed method is shown below:

$$
\left[\begin{array}{lllll}
0 & 0 & 1 & 0 & 0 \\
0 & 0 & 1 & 0 & 0 \\
1 & 1 & 1 & 1 & 1 \\
0 & 0 & 1 & 0 & 0 \\
0 & 0 & 1 & 0 & 0
\end{array}\right]
$$

After dilation enhanced 2D blob is obtained. For fast and extraction of the 'motion' feature, enhancement is done. [2], [5], [11] easy

The blob extraction is shown below:

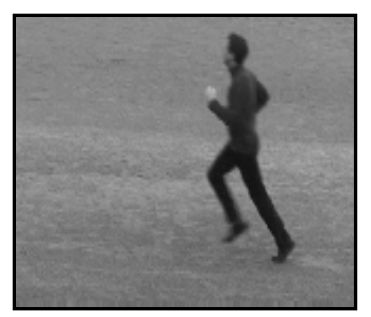

Input frame

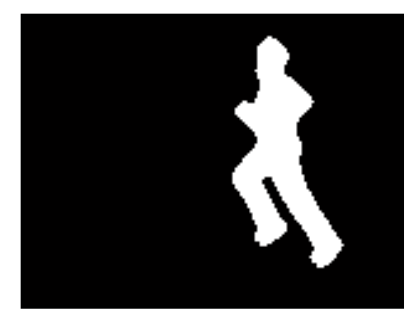

dilated frame
Fig-2: Blob extraction

\subsection{Feature Extraction}

After segmentation process, the next stage is feature extraction. In this stage mid-level feature 'motion' is extracted from the blob. Since the human action can be effectively characterized by motion rather than other features such as color, texture or shape, 'motion' feature is extracted from the blob.We use optical flow to estimate motion. Optical flow estimates the direction and speed of moving object from one video frame to another. There are two methods to find the Optical flow Horn-Schunck or Lucas-Kanade method. For floating point input HornSchunck method is used and for fixed point input LucasKanade method is used. We use Lucas-Kanade method to find the optical flow. [1], [7], [8]

The optical flow of input video frame is shown below:

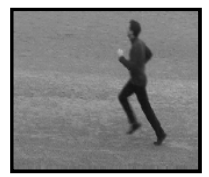

Input frame

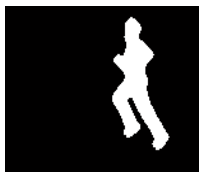

dilated frame

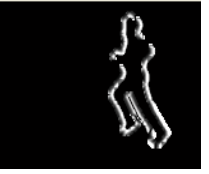

optical flow
Fig-3: Feature Extraction

\subsection{Action Recognition}

This is the last stage of action recognition. For recognition, we use k Nearest Neighbor (kNN). [1], [3], [4], [6]

$\mathrm{kNN}$ is a standard classifier which is mostly used for action recognition because it does not require any learning process and also it is invariant against view-point, spatial and temporal variations.

Before classification using $\mathrm{kNN}$, the proposed method computes the following steps:

i) First the centroid of the connected region in the optical flow is computed. This is called Blob analysis.

ii) The dimension of Blob analysis is very large; hence to reduce its dimension Principal Component Analysis (PCA) is done. PCA is a technique which reduces the dimension of large data sets.

iii) Then covariance matrix of PCA is found.

iv) Then eigenvalues (EVA) of the covariance matrix are found.EVA measure the magnitude of the corresponding relative motion. This is called the training data for classification.

v) Then the nearest neighbor in the training data is searched using the distance metric 'Euclidean distance' of kNN.

vi) After $\mathrm{k}-\mathrm{Nearest}$ Neighbor $(\mathrm{kNN})$ search, they are classified using kNN classifier. If the 1-NN is obtained for each action in the dataset then it means that the action is recognized.

vii) The result is also plotted using function 'gscatter' to observe the classification. 


\section{FLOWCHART OF THE PROPOSED \\ METHOD}
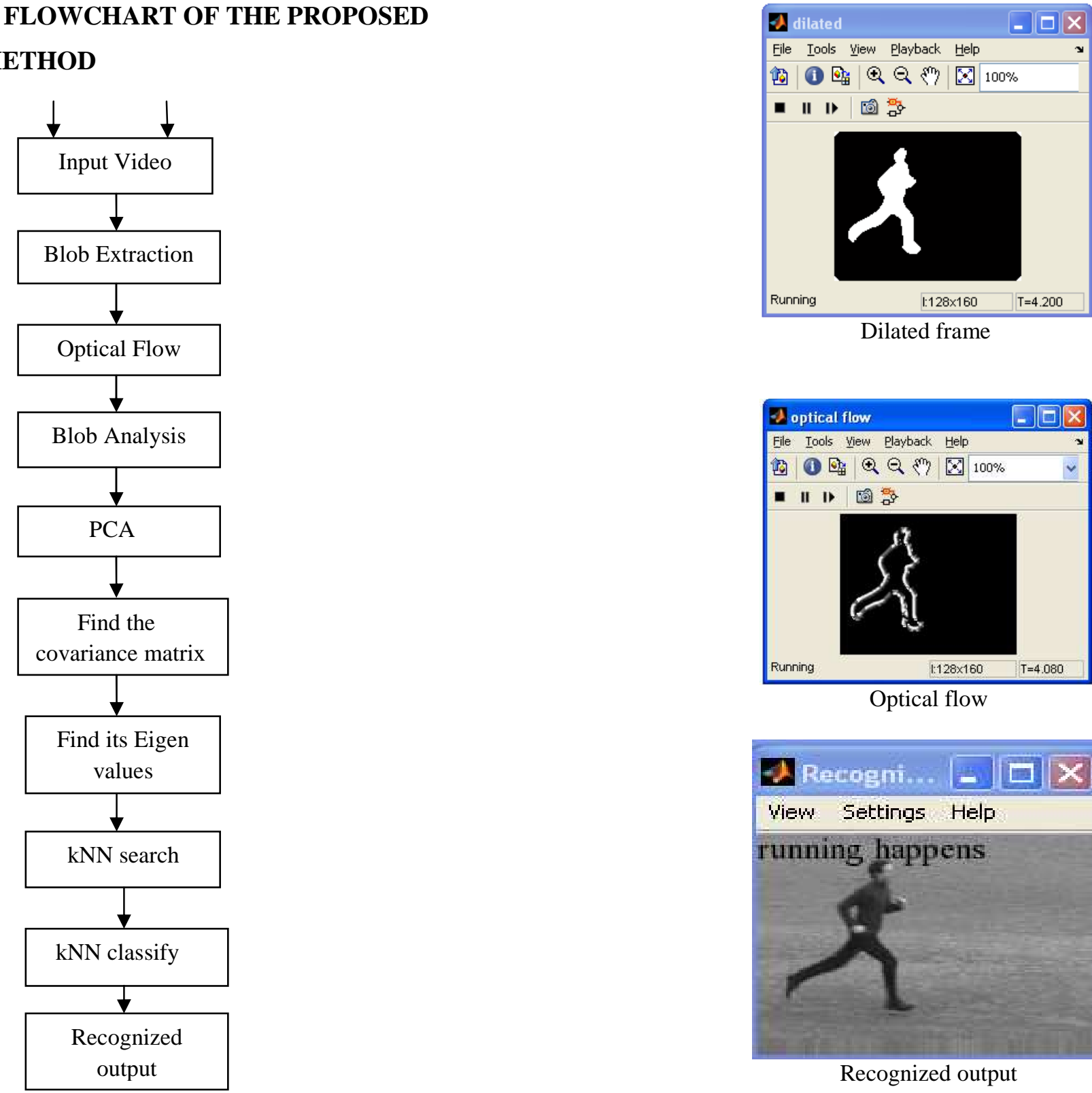

Dilated frame
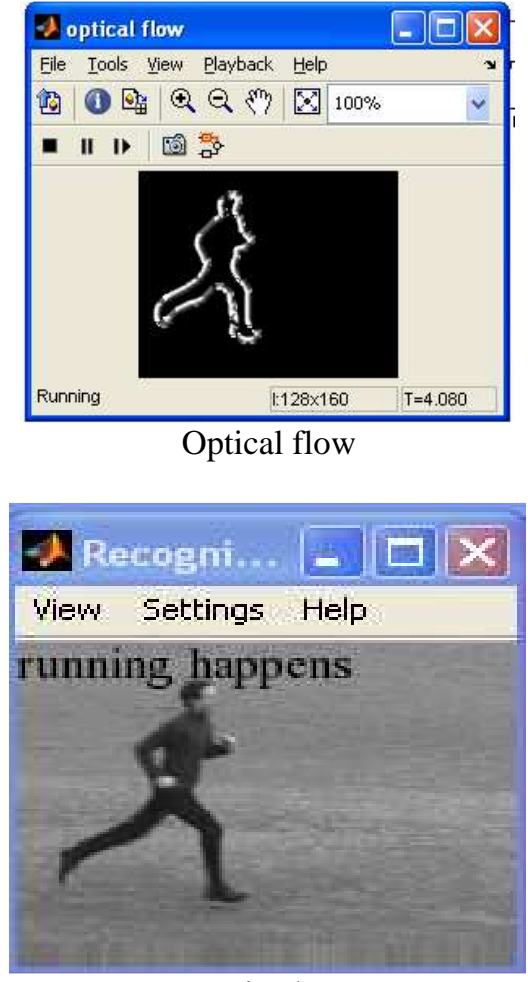

Recognized output

\section{RESULTS}
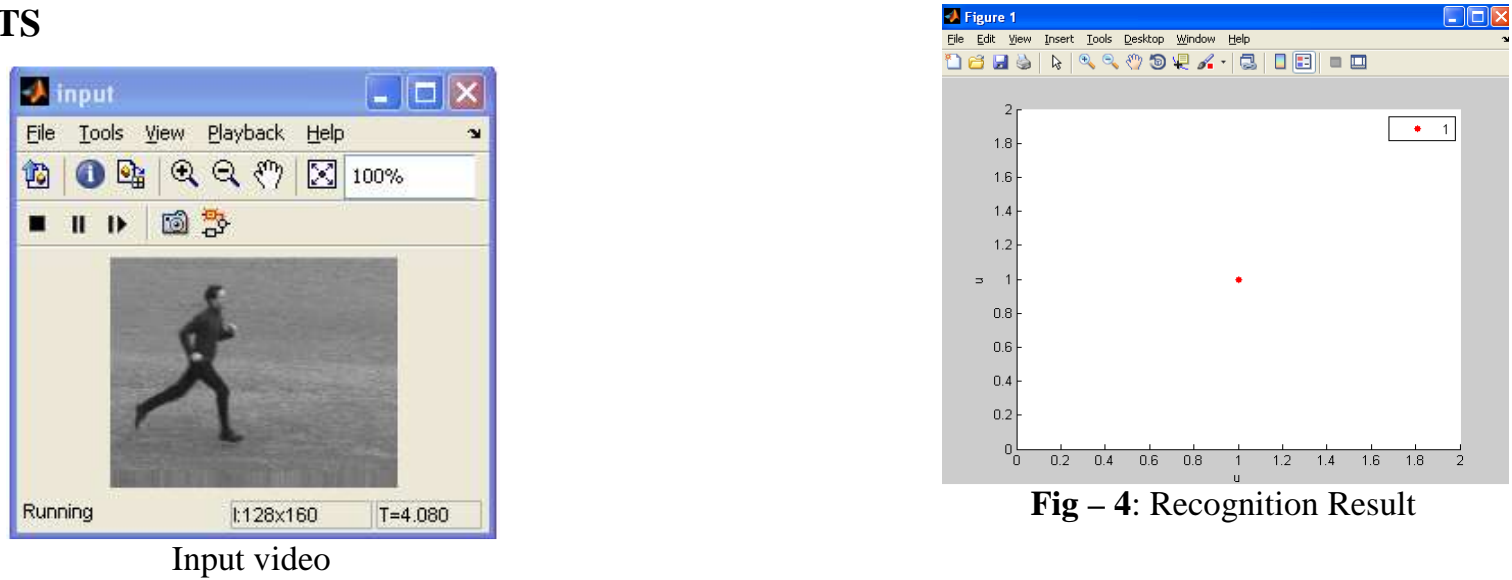

Fig - 4: Recognition Result 


\section{DISCUSSION}

By using the proposed method the accuracy of recognition are shown below:

Table 1.Recognition using KTH Dataset

\begin{tabular}{|l|c|c|c|}
\hline $\begin{array}{l}\text { Type of } \\
\text { sequence }\end{array}$ & $\begin{array}{l}\text { Total } \\
\text { seq }\end{array}$ & $\begin{array}{l}\text { Correctly } \\
\text { Recognized }\end{array}$ & In \% \\
\hline walking & 10 & 10 & 100 \\
\hline running & 10 & & 100 \\
\hline hand waving & 10 & 10 & 100 \\
\hline handclapping & 10 & 10 & 100 \\
\hline Boxing & 10 & 10 & 100 \\
\hline jogging & 10 & 10 & 100 \\
\hline & $\Sigma=60$ & $\sum=60$ & Avg =100 \\
\hline
\end{tabular}

Average \% of accuracy using KTH dataset is $100 \%$.

Table 2.Recognition using Weizmann Dataset

\begin{tabular}{|c|c|c|c|}
\hline $\begin{array}{l}\text { Type of } \\
\text { sequence }\end{array}$ & Total seq & $\begin{array}{l}\text { Correctly } \\
\text { Recognized }\end{array}$ & In \% \\
\hline walk & 9 & 9 & 100 \\
\hline run & 9 & 9 & 100 \\
\hline jack & 9 & 9 & 100 \\
\hline skip & 9 & 9 & 100 \\
\hline side & 9 & 9 & 100 \\
\hline bend & 9 & 9 & 100 \\
\hline jump & 9 & 9 & 100 \\
\hline pjump & 9 & 9 & 100 \\
\hline wave 1 & 9 & 9 & 100 \\
\hline wave 2 & 9 & 9 & 100 \\
\hline & $\Sigma=90$ & $\Sigma=90$ & Avg $=100$ \\
\hline
\end{tabular}

Average $\%$ of accuracy using Weizmann dataset is $100 \%$.

\section{CONCLUSION}

This paper has presented a motion-based approach for action recognition. It has used 2D blob as low-level feature and extracts mid-level feature 'motion' from the blob using the method Lucas Kanade of optical flow. The motion features so obtained are classified using $\mathrm{kNN}$ classifier. The advantage of using $\mathrm{kNN}$ is that it does not require any learning process and also it is invariant against view-point, temporal and spatial variations; hence its accuracy is good. The average \% of accuracy using the proposed method is $100 \%$ on Weizmann and KTH datasets.

\section{REFERENCES}

[1] S. Hari Kumar, P.Sivaprakash, "New Approach for Action Recognition Using Motion based Features", Proceedings of 2013 IEEE Conference on Information and Communication Technologies (ICT 2013), pp.1247-1252.

[2] Hetal Shah, N. C. Chauhan, "Recognition of Human Actions in Video", International Journal on Recent and Innovation Trends in Computing and Communication (IJRITCC) May 2013, ISSN 2321 -8169Volume 1,Issue 5, pp. $489-493$.

[3] Xiaodong Yang and YingLi Tian, "Eigen Joints-based Action Recognition Using Naïve-Bayes-Nearest-Neighbor", 2012 IEEE, pp.14-19.
[4] Mi Zhang Alexander A. Sawchuk, "Motion PrimitiveBased Human Activity Recognition Using a Bag-ofFeatures Approach”, IHI'12, January 28-30, 2012, Miami, Florida, USA.

[5] Muhammad Hameed Siddiqi, Muhammad Fahim, Sungyoung Lee, Young-Koo Lee, “ Human Activity Recognition Based on Morphological Dilation followed by Watershed Transformation Method ", 2010 International Conference on Electronics and Information Engineering (ICEIE 2010), Volume 2, 2010 IEEE,V2 433-V2 437.

[6] Ronald Poppe, "A survey on vision-based human action recognition", Science Direct Image and Vision Computing 28 (2010) 976-990.

[7] Mohiuddin Ahmad, Seong-Whan Lee, "Human action recognition using shape and CLG-(Combined local-global) motion flow from multi-view image sequences", Science Direct Pattern Recognition 41 (2008), 2237 - 2252.

[8] Mohiuddin Ahmad and Seong-Whan Lee, "HMM-based Human Action Recognition Using Multiview Image Sequences", Proceedings of the 18th International Conference on Pattern Recognition (ICPR'06), 2006 IEEE.

[9] Moshe Blank, Lena Gorelick, Eli Shechtman, Michal Irani, Ronen Basri, “ Actions as space-time shapes", Proceedings of the International Conference On Computer Vision (ICCV'05), vol. 2, Beijing, China, October 2005, pp. 1395- 1402 .

[10] O. Masoud and N. Papanikolopoulos, "A method for human action recognition,” IVC, Vol. 21, 2003, pp.729-743.

[11] J. K. Aggarwal and Q. Cai, "Human Motion Analysis: A Review", idealibrary: Computer Vision and Image Understanding,Vol. 73, No. 3, March 1999, pp. 428-440.

[12] http://www.nada.kth.se/cvap/actions 